\title{
¿ES LA LÓGICA ANALÍTICA O SINTÉTICA?
}

\author{
Amparo DIÉZ MARTÍNEZ* \\ Dpto. de Lógica, Historia y Filosofía de la Ciencia. UNED
}

RESUMEN: La tesis que se defiende en este trabajo es que la lógica (de Primer Orden o superior) tiene las caracteristicas que Kant reconoció en las verdades sintéticas a priori: son necesarias, anteriores a la experiencia, pero tambien informativas $y$, por tanto, dependientes de la sensibilidad. Se destacan y comentan, en primer lugar, algunos textos kantianos sobre lo analítico y lo sintético. En segundo lugar, se examina de qué está hablando Kant cuando afirma que "la lógica es analítica" y con qué significado (distinto) es utilizada esa misma expresión por parte de Frege. Con estos elementos a la vista, pasamos a defender la idea, original de Hintikka, de que algunas verdades de la lógica clasica son sintéticas, su prueba exige el concurso de la intuición pura.

\section{Introducción}

En este artículo no discutiré si es posible, adecuado o interesante distinguir entre verdades analíticas y verdades sintéticas, ni entraré, por tanto, en la discusión clásica, que comienza con el Positivismo Lógico y llega hasta nuestros días principalmente en las obras de Quine, Kripke y Putnam, sobre sinonimia, nombres propios y descripciones en mundos posibles, cuestiones epistemológicas, compromisos ontológicos, etc. El problema que nos ocupa no es el de la distinción entre lo analítico y lo sintético en general ni, menos aún, en el lenguaje natural. El único objetivo que se plantea en este trabajo es el de tratar de responder a la pregunta que le da título, atendiendo, en primer lugar, a la descripción kantiana de las características de esas dos nociones y, en segundo lugar, a su lógica general, en contraposición con la lógica formal presentada más tarde por Frege y examinando también qué significa para Frege «analíticow. En este contexto de la lógica formal clásica de

" adiez@psof.uned.es 
predicados es donde defenderé que algunas de las verdades lógicas, siendo necesarias y a priori, son también sintéticas. Para ello acudo al argumento dado por Hintikka: al aplicar la regla de eliminación del cuantificador existencial "parecemos anticipar la existencia de un individuo con ciertas propiedades antes de que la experiencia nos lo haya proporcionado» (Hintikka 1976: 145).

\section{La distinción kantiana entre analítico y sintético}

Las tres nociones más utilizadas por Kant para decirnos qué entiende por analítico y qué por sintético, son las de identidad, información e intuición.

«Se llaman proposiciones analiticas aquellas cuya certeza se basa en la identidad de los conceptos (del predicado con la noción del sujeto). Proposiciones cuya verdad no se funda en la identidad de los conceptos han de ser denominadas sintéticas. Observ. 1 . Todo $\mathrm{x}$, al cual conviene el concepto de cuerpo $(\mathrm{a}+\mathrm{b})$, le conviene también la extensión (b), -es un ejemplo de proposición analítica- Todo $x$ al cual conviene el concepto de cuerpo $(a+$ b), le conviene también la atracción (c) —es un ejemplo de proposición sintética-. Las proposiciones sintéticas incrementan el conocimiento materialiter; las analíticas meramente formaliter. Aquellas contienen determinaciones, éstas solamente predicados lógicos" (Kant, 2000: 157).

En esta definición podemos ya observar que el contexto lógico-lingüístico de su discurso es uno en el que las unidades de significación están estructuradas en forma de sujeto y predicado. Ambas funciones, la de sujeto y la de predicado, son realizadas por conceptos y las relaciones que concibe entre ellos son relaciones de inclusión o exclusión ${ }^{1}$. Si la conexión entre el sujeto y el predicado es pensada mediante la identidad, estamos ante un juicio analítico: el concepto-predicado está contenido en el concepto-sujeto. En los juicios sintéticos, el concepto-predicado ofrece información que no estaba contenida en el concepto-sujeto, gracias a las determinaciones (intuiciones) que contienen.

Así, los juicios analíticos son meramente explicativos, el predicado no añade nada, tan sólo hace más distinto el concepto-sujeto, lo hace más claro, pero mediante la distinción, los conceptos mejoran sólo en cuanto a la forma, no en cuanto al contenido. En cambio, los juicios sintéticos son ampliativos de conocimiento.

${ }^{1}$ Kant 2000, Refl 3045: "Juzgar: representar un concepto como contenido en otro». 
«Los juicios analíticos (los afirmativos) son, pues, aquellos en los cuales el enlace del predicado con el sujeto es pensado mediante identidad. Aquellos, empero, en que este enlace es pensado sin identidad, deben llamarse juicios sintéticos. Los primeros pudieran también llamarse juicios de explicación, los segundos juicios de ampliación" (Kant, 1982: 31).

Está claro que los juicios analíticos se producen sin recurso a la experiencia y que los que resultan de la experiencia son sintéticos. La pregunta es si efectivamente se producen conocimientos intuitivos sin recurrir a la experiencia, es decir, a priori. ¿Cómo puede haber una representación sensible sin impresiones sensoriales? Según Kant, podemos producirla a priori:

"El primero que demostró el triángulo isósceles (háyase llamado Thales o como se quiera), percibió una luz nueva; pues encontró que no tenía que inquirir lo que veía en la figura ni aun en el mero concepto de ella y por decirlo así aprender de ella sus propiedades, sino que tenía que producirla, por medio de lo que, según conceptos, él mismo había pensado y expuesto en ella a priori (por construcción), y que para saber seguramente algo a priori, no debía atribuir nada a la cosa, a no ser lo que se sigue necesariamente de aquello que él mismo, conformemente a su concepto, hubiese puesto en ella" (Kant, 1982: 12).

Esta es una cuarta característica que distingue los juicios sintéticos a priori de los analíticos: los primeros son constructivos de determinaciones de los conceptos en la sensibilidad. La ampliación de conocimientos, sea a priori o a posteriori, necesita el concurso de la sensibilidad, única facultad de las intuiciones.

"Construimos conceptos cuando los exponemos en la intuición a priori, sin experiencia, o cuando exponemos en la intuición el objeto que corresponde a nuestro concepto del mismon (Kant, 2000: 91).

Si el juicio depende de la construcción de una intuición (de la exposición en la intuición de un objeto - un caso- que ejemplifica el concepto), decimos que es sintético.

Por último, hay otro criterio kantiano de analiticidad que no se cita con tanta frecuencia como los anteriores y que, como veremos, es importante para la lógica: una afirmación es analítica, si su negación contiene una contradicción.

"Debemos dar al principio de contradicción el valor de principio universal y plenamente suficiente de todo conocimiento analitico" (Kant, 1982: 102).

Resumiendo, un juicio analítico es, al igual que algunos de los enunciados sintéticos, a priori (anterior a la experiencia), pero, a diferencia de ellos, es mera- 
mente explicativo, no ampliativo de conocimientos, no constructivo de intuiciones y tautológico.

\section{La supuesta analiticidad de la lógica: lógica general kantiana y lógica fregeana}

Kant define la lógica general como la ciencia de las leyes necesarias del pensamiento, sin las cuales no podría haber uso alguno del entendimiento y de la razón ${ }^{2}$. La lógica es meramente formal ${ }^{3}$, abstrae de todo contenido, de cualquier objeto del conocimiento y de sus diferencias, se ocupa sólo de la forma del pensar, de las leyes que gobiernan el uso del entendimiento.

“Esta ciencia de las leyes necesarias del entendimiento y de la razón en general, o - lo que es lo mismo- de la mera forma del pensamiento en general, lo denominamos, pues, lógicas (Kant, 2000: 80).

Siendo el entendimiento la facultad de concebir reglas en general, la facultad de someter a leyes las representaciones, también él está sujeto a leyes, pues todo en la naturaleza se produce según reglas. Algunas de las reglas del pensar son necesarias, otras son contingentes. Necesarias son aquellas sin las cuales no tendría lugar ningún uso del entendimiento, contingentes aquellas según las cuales tienen lugar ciertos usos particulares, por ejemplo, el uso matemático, pues es contingente pensar tal o cual objeto. Las necesarias son independientes de la experiencia, ya que expresan las condiciones de todo uso del entendimiento, sea puro o experimental, su estudio ha de ser a priori y puramente formal, sin recurso a contenidos empíricos ${ }^{4} . ~ ¿$ Recurre la lógica a algún contenido no empírico, uno que requiera sólo intuiciones puras? Según Kant, tampoco. La validez de un argumento deductivo no depende en ningún sentido del significado de las palabras, sino únicamente del modo de composición, la validez se predica de una estructura formal vacía de contenido.

Además, la lógica no trata de cómo tiene lugar empíricamente un proceso cognitivo, ni se ocupa de frecuencias estadísticas sobre disposiciones lingüísticas; a la lógica le interesa únicamente cómo se debe pensar, si estamos interesados en la verdad. Es decir, la lógica no es psicología.

\footnotetext{
${ }^{2}$ Cfr. Kant, 1982: 59 y 2000: 84-5.

${ }^{3}$ Cfr. Kant, 1982, prólogo.

${ }^{4}$ Cfr. Kant, 2000: 78-9.
} 
*La lógica es (...) una ciencia a priori de las leyes necesarias del pensamiento (...), una ciencia, por tanto, del uso correcto del entendimiento y de la razón en general, pero no en sentido subjetivo, es decir, no segrin principios empiricos (psicologicos): cómo piensa el entendimiento, sino en sentido objetivo, es decir, según principios a priori: cómo debe pensar» (Kant, 2000: 84).

Frege suscribe completamente este texto y nos aclara qué significa ese "cómo debe pensarw: las leyes del pensar son leyes de la verdad y no leyes del tener por verdadero. Ambos se esfuerzan por dejar claro que son leyes normativas, pero no en el mismo sentido que las naturales, pues entonces serían leyes psicológicas. Una ley lógica no dice "uno debe pensar que todo objeto es idéntico consigo mismo", ni wen un tiempo, al hombre le fue imposible pensar que algún objeto no es idéntico consigo mismo", sino "todo objeto es idéntico consigo mismo" $(\forall \mathrm{x} x=\mathrm{x})$. Las leyes lógicas reflejan la legalidad del entendimiento y la razón, las condiciones necesarias del pensamiento correcto en general. Frege nos avisa del riesgo que supone asimilar demasiado esta legalidad a la legalidad de la naturaleza:

"Es funesto aquí el sentido de la palabra «ley». En un sentido afirma lo que es, en el otro prescribe lo que debe ser. Solamente en este último sentido pueden ser llamadas las leyes lógicas leyes del pensamiento, al fijar el modo como hay que pensar (...). Pero el término "ley del pensamiento" induce a la opinión errónea de que estas leyes rigen el pensamiento del mismo modo que las leyes naturales los sucesos del mundo exterior. En tal caso no pueden ser otra cosa que leyes psicológicas; pues el pensamiento es un proceso mental. (...) Estas leyes no están con nuestro pensamiento en la misma relación que las leyes gramaticales con el lenguaje de modo que fueran expresión de la naturaleza de nuestro pensamiento humano y cambiasen con él" (Frege, 1984: 138-40).

Las leyes lógicas expresan cómo se debe pensar, por quien sea, donde sea y cuando sea, si se quiere alcanzar la verdad o conservarla cuando se tiene. A diferencia de Kant, Frege concibe la lógica no como un mero cálculo vacío de contenidos, sino como un lenguaje, con contenido propio. La lógica siendo general (universalmente aplicable), no es meramente formal. En el lenguaje lógico se presupone al menos el tipo de cosas que pueden ser candidatos para interpretar las expresiones no lógicas. Además, las expresiones lógicas tienen un significado bien definido dentro del sistema en el que son usadas. La lógica es un lenguaje capaz de expresar contenidos.

«Desde el principio tuve en mente la expresion de un contenido (...), una lingua characterica en primer lugar para las matemáticas" (Frege, 1979: 12). 
Kant necesita insistir en la formalidad de la lógica general para distinguirla de la lógica transcendentals:

"La lógica general hace abstracción, como hemos visto, de todo contenido del conocimiento, es decir, de toda referencia del conocimiento al objeto y considera solamente la forma lógica en la relación de los conocimientos entre sí, es decir, la forma del pensamiento en general. Ahora bien, así como hay intuiciones puras y empíricas (según demuestra la estética transcendental), así también podría hallarse una distinción entre un pensar puro y un pensar empírico de los objetos. En este caso, habría una lógica en la cual no se hiciera abstracción de todo contenido del conocimiento" (Kant, 1982: 60).

Kant y Frege coinciden en que las leyes lógicas son leyes del pensar en general y son las leyes formales de la verdad, son leyes necesarias y universales, pero para Kant la lógica es un canon (un sistema de reglas) mientras que para Frege es un lenguaje, con una gramática y una semántica, un sistema de verdades (un sistema de leyes).

«La ciencia que contiene estas reglas universales y necesarias es meramente una ciencia de la forma de nuestro conocimiento intelectual o del pensamiento. Y nosotros podemos formarnos la idea de la posibilidad de una ciencia tal, como de una gramática general, que no contiene más que la mera forma de la lengua en general, sin palabras, que pertenecen únicamente a la materia de la lengua" (Kant, 2000: 80).

Kant no conoce el lenguaje formal lógico, sino que tiene presente sólo un lenguaje normalizado como el de la silogística. Si hubiera conocido el desarrollo del lenguaje formal con cuantificadores, quizás hubiera dicho que esta lógica no es ya una lógica general, sino una especial, una que no abstrae completamente de todo contenido.

Si la nueva lógica es un lenguaje y no sólo una gramática, quizás pueda, e incluso necesite, en algún momento recurrir a la construcción de intuiciones puras, como ocurre, por ejemplo, con la geometría.

${ }^{5}$ La lógica transcendental se aplica a un objeto especial: el entendimiento, se ocupa del origen, extensión, etc. de los conceptos a priori, no se limita a conocer las leyes del entendimiento, sino que conoce que tales representaciones no tienen origen empírico y que se refieren a priori a objetos de la experiencia posible. 
«En un teorema geométrico no se hace mención de la línea auxiliar, que a lo mejor se ha utilizado para la prueba. Quizá son posibles varias, por ejemplo, cuando se puede escoger arbitrariamente un punto. Pero por mucho que podamos prescindir de cada línea auxiliar en particular, no obstante la fuerza de la prueba depende de que se pueda trazar una línea de las características exigidas" (Frege, 1973: \$102).

No se entiende bien cómo Frege no vio que muchas pruebas lógicas dependen precisamente de suponer algún objeto que cumple ciertas propiedades, podemos prescindir de cada objeto auxiliar en particular, pero la prueba depende de que se pueda poner (construir) un objeto con las características exigidas para hacer un cálculo provisional. Frege reconoce el gran mérito de Kant al haber señalado que la geometría requiere de la intuición pura del espacio y es, por tanto, sintética, pero su afán por reducir la aritmética a lógica y no contaminar a ésta con nada que pertenezca a la psicología, le conduce a modificar la noción de analítico, que pasa a significar reducible a lógica. Lo que Frege quiere impedir al decir que la aritmética es analítica, es que se piense que pueda ser a posteriori, que pueda depender de intuiciones a posteriori, es decir, de fenómenos, pues la aritmética no se aplica únicamente a lo intuible, sino que rige el dominio de lo numerable, que no es sino todo lo pensable (a priori o a posteriori).

Tras el descubrimiento de la paradoja que la ley $\mathrm{V}$ produce en su sistema, abandona la defensa del logicismo, pero no revisa la supuesta analiticidad de la lógica. La analiticidad es ahora aplicada por Frege al tipo de prueba que requiera cada verdad: si exige únicamente definiciones y leyes lógicas, la verdad es analítica, si necesitamos hacer uso de definiciones y leyes de otras ciencias, la verdad es sintética (a priori o a posteriori). Esta noción de analiticidad conserva algunas de las características señaladas por Kant: 1) la lógica no necesita para sus pruebas la construcción de objeto alguno en la intuición, y 2) la lógica se basa en la identidad y la no contradicción; pero ya no pertenece a ella el ser meramente explicativa. Naturalmente si la aritmética es fuente de conocimiento, como también afirma Frege, y si la lógica ha de ser un lenguaje capaz de expresar las verdades aritméticas, también la lógica deberá ser fuente de conocimiento, la lógica es una ciencia ampliativa, aunque analítica.

Definir los juicios analíticos como aquellos que no dicen nada en el predicado que no hubiera sido pensado ya en el sujeto, aunque no tan claramente, no con la misma consciencia, tiene muy difícil aplicación a los juicios existenciales sobre un predicado monádico, por ejemplo, que aparecen en la nueva lógica fre- 
geana. Esa analiticidad, aplicada a argumentos deductivos lógicamente correctos, significaría que nada puede ser dicho en la conclusión que no haya sido afirmado ya en el conjunto de las premisas; si acaso, podría haber solamente un avance psicológico, quizás si hubiéramos empleado más tiempo y prestado la atención suficiente a lo que se dice en las premisas, habríamos podido ver cómo está contenida en ellas la conclusión. Sin embargo, es sencillamente falso que al conocer un conjunto de premisas, conocemos (podemos conocer en todos los casos) el conjunto de todas sus consecuencias lógicas.

Pero en lugar de afirmar que la lógica es sintética por ser informativa, Frege prefiere introducir las modificaciones oportunas para hacer compatibles las dos nociones de analitico y ampliación de conocimiento.

«Kant subestimó claramente el valor de los juicios analíticos (...), si bien parece que también barruntó el concepto más amplio aquí utilizado (...). Kant estaba pensando, al hablar asi, en el caso del juicio afirmativo universal. Entonces puede hablarse de un concepto de sujeto y preguntarse si el concepto de predicado está contenido en él (...). Pero ¿qué ocurre cuando el sujeto es un objeto único? ¿Y qué cuando se trata de un juicio existencial? (...). Las definiciones conceptuales más útiles son las que marcan líneas fronterizas que aún no habian sido trazadas en absoluto. Lo que pueda inferirse a partir de ellas no puede determinarse de antemano; en este caso no se vuelve simplemente a sacar de la caja lo que se habta metido en ella. Las conclusiones que sacamos de este tipo de definición aumentan nuestro conocimiento $y_{\text {, }}$ siguiendo a Kant, deberían ser consideradas en consecuencia como sintéticas; no obstante, pueden ser demostradas de modo puramente lógico y, por lo tanto, son analíticas. Están contenidas de hecho en las definiciones, pero lo están como la planta en la semilla, no como las vigas en la casas (Frege, 1973: $\$ 88$, subrayado mí) 6 .

En una argumentación correcta la conclusión se sigue con necesidad lógica del conjunto de las premisas (por eso llama analítico a todo el proceso), pero no la teníamos antes de hacer el cálculo. En la propuesta fregeana se reserva como exclusiva del conocimiento sintético la dependencia necesaria de la intuición (sensibilidad), y se deja que su otra característica, su ampliación de información, pueda ser aplicada también a juicios analíticos.

${ }^{6}$ Kant sólo concibe dos vías para la formación de conceptos: mediante análisis del concepto en sus notas o mediante síntesis de notas: no es lo mismo whacer distinto un conceptom, descomponerlo en sus notas, que whacer un concepto distinto", sumar notas para formar un nuevo concepto. Cfr. Kant 2000: 122-3. 
En la lógica formal cuantificacional se pueden escribir fórmulas como esta: $\forall \mathbf{x}$ $\forall y(R x y \rightarrow \exists z(R x z \wedge R z y))$, la cual puede recibir una interpretación que exprese la divisibilidad infinita de una línea: uentre cualesquiera dos puntos de una recta existe un tercer puntom. Si Kant hubiera conocido este lenguaje chabría dicho que esta es una ciencia sintética a priori, dado que los objetos sólo pueden ser pensados mediante intuiciones, o no?, lo que se dice en la conclusión de un argumento válido ¿está afirmado en las premisas o se halla fuera de ellas, aunque necesariamente conectado a lo que se dice en ellas?

\section{Pruebas lógicas. La tesis de Hintikka}

Ambos, Frege y Kant, justifican el carácter sintético de las verdades geométricas poniendo de manifiesto que en sus pruebas necesitamos recurrir a la intuición. Si esa es una condición suficiente, también ambos deberían reconocer el carácter sintético de la lógica de Primer Orden, algunas de cuyas verdades dependen necesariamente de la construcción de una intuición a priori, hemos de salir del concepto e ir a la intuición que puede instanciarlo, es decir, construir un representante del concepto, determinar el concepto a priori.

La lógica nos permite capturar la forma de los razonamientos deductivos, y la validez de los esquemas lógicos no depende de la interpretación concreta que reciban sus expresiones no lógicas. En este sentido, razonar deductivamente es calcular, la lógica es un cálculo (la forma) del razonamiento deductivo, pero el significado de los cuantificadores reiterados sólo se puede captar mediante alguna construcción en la intuición pura, los cuantificadores no representan únicamente conceptos, sino conceptos ligados a intuiciones, y así, la lógica de Primer Orden sería más bien, como la matemática, sintética.

Si toda argumentación correcta puede ser transformada en una tautología y esto se entiende en el sentido de que la conclusión no nos da nada que no estuviera puesto en las premisas, debemos preguntarnos cuál es la utilidad de la argumentación deductiva y si es cierto que la novedad que pudiera presentarse en la conclusión no puede ser nada objetivo, sino únicamente una novedad psicológica. Una tautologia, se dice, es un esquema lógico vacío de contenido, que no dice nada sobre el mundo. Pero muchas tautologías necesitan prueba, no son autoevidentes. En muchas ocasiones, cuando presentamos una prueba de la validez de un argumento estamos ofreciendo información objetiva en dos sentidos: 1) la información de que efectivamente el argumento es correcto, ofrecemos una verdad lógica: existe una relación de consecuencia lógica entre las premisas y la con- 
clusión, el argumento vale por tener una determinada estructura lógica; y 2) la información de que es necesario que exista una cierta verdad empírica, si existen otras verdades empíricas, nos dice algo sobre el mundo: cómo son las cosas en un mundo del que ya sabemos algo.

Comparto la tesis de Hintikka de que las inferencias lógicamente correctas pueden darnos algo objetivamente nuevo en la conclusión, aunque no comparto algunas de sus justificaciones, que me parecen demasiado psicologistas. Según Hintikka, para Dios todas las verdades lógicas serían analíticas porque podría considerar instantáneamente todos los objetos auxiliares necesitados en las premisas, mientras que el sujeto humano necesita introducir esos objetos en el tránsito desde las premisas hasta la conclusión. Mi posición, en cambio, es más bien que hemos de medir la analiticidad por el modo de producción de la conclusión. No se trata de que el sujeto humano es incapaz de ver la conclusión en las premisas, sino de que, en algunos casos, la conclusión sencillamente no está en las premisas, sino que únicamente se sigue necesariamente de ellas. Aunque esa necesidad parece indicar analiticidad por tratarse, en principio, de una necesidad formal, hemos de comprobar las características de esa formalidad en los distintos procesos efectivos de prueba.

Ni siquiera Dios puede computar lo no computable, ni puede en todos los casos ver la conclusión de un argumento sin considerar algunas posibilidades objetuales, tampoco para él son analíticas algunas verdades, a menos que lo que queramos decir es simplemente que "todo sucede según reglas"; pero si hacemos eso borramos ya no sólo la distinción entre aritmética y lógica, como quiso hacer Frege, sino también la distinción entre geometría y lógica. La cuestión es si la conclusión está contenida en las premisas como las vigas en una casa o como la planta en la semilla, si el "ver la conclusión en las premisas" se asemeja al «ver 12 aristas en la figura que resulta de tres pares de planos perpendiculares entre sí» o más bien a "ver que $a$ está contenido en $(a+b)$ ). No importa si se tiene capacidad o no para considerar instantáneamente todos los objetos auxiliares necesitados en el cálculo, lo que hace que una verdad sea sintética es la necesidad de considerar tales objetos auxiliares.

Kant y Frege insistieron en la idea de que una relación de consecuencia es lógica cuando no puede suceder que las premisas sean verdaderas mientras la conclusión es falsa, o, lo que es lo mismo, la verdad de las premisas junto con la falsedad de la conclusión arroja una contradicción, dicho de otro modo, negar que la conclusión es implicada por las premisas es contradictorio. 
El cálculo mediante árboles semánticos, por ejemplo, nos ofrece el resultado buscado: la información de que hay mundos o situaciones donde las fórmulas examinadas (premisas y negación de la conclusión) pueden ser todas ellas verdaderas a la vez o que no hay ningún mundo así; es decir, que la verdad de la negación de la conclusión es compatible o no con la verdad de las premisas.

Podríamos ver si hay o no contradicción sin recurrir a un medio físico (por ejemplo, un papel y un lápiz) donde realizar el cálculo, pero no sin transitar en algún momento por alguna intuición a priori. Consideremos el siguiente esquema argumental:

$$
\frac{\exists x \operatorname{Px} \wedge \exists y Q y}{\exists x(P x \wedge Q x)}
$$

Su árbol semántico es el siguiente:

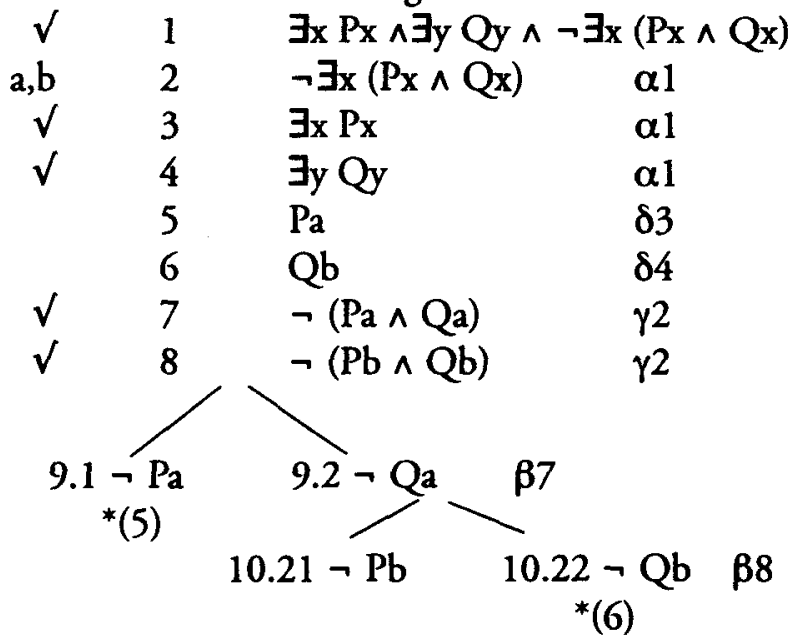

El árbol está terminado y la rama 1 - 10.21 está abierta, indicándonos un mundo posible donde la premisa puede recibir una interpretación verdadera y la conclusión una falsa sin caer en contradicción, un mundo donde hay dos objetos distintos. En la línea 5 hemos necesitado introducir un objeto como caso de una afirmación existencial y otro distinto en la línea 6 . Necesitamos introducir esos dos objetos nuevos para poder alcanzar el conocimiento de que ese esquema argumental no es válido, que la fórmula escrita en la línea 1 es satisfacible.

Pero alguien podría protestar: jese no es un ejemplo de verdad analítica, no es una verdad lógica!, ¡de lo que se trata es de si las tautologias, es decir, las verda- 
des lógicas son analíticas o no! Antes de seguir avanzando en esta dirección, veamos qué noción de analiticidad utiliza Hintikka:

«Un paso analítico de argumento no puede llevarnos de la existencia de un individuo a la existencia de diferentes individuos" (Hintikka 1976: 163).

Este es, según Hintikka, el sentido que Kant le quiso dar y cualquier argumento que viole ese principio no es sino un método sintético de razonar.

Hintikka distingue tres sentidos principales de la noción de analiticidad aplicada a los esquemas argumentales lógicos: 1) como una relación entre los conceptos presentes en la conclusión y los conceptos puestos en juego en el conjunto de las premisas, 2) mediante la noción lógica de subfórmula -la conclusión es una subfórmula de la conjunción de las premisas-, y 3) ontológicamente: el paso de las premisas a la conclusión no nos obliga a introducir nuevos individuos en nuestro universo de discurso. Centrándose en el tercer sentido de analiticidad, precisa una posible reconstrucción de la noción kantiana del siguiente modo:

Un paso de argumento (válido) es analítico si no introduce nuevos individuos en la discusión.

- Un argumento analítico no puede llevar de la existencia de un individuo a la existencia de un individuo diferente.

- Un paso de argumento (válido) es analítico si no aumenta el número de individuos que estamos considerando en su relación mutua.

- Un paso de argumento (válido) es analítico si el grado de la conclusión no es mayor que el grado de al menos una de las premisas. Un argumento es analítico si todos sus pasos son analíticos en ese sentido.

- Una prueba (válida) de la oración $F_{1}$ a partir de $F_{0}$ es analítica si ninguna de las oraciones que figuran como etapas intermedias de esta prueba tiene un grado superior a $F_{0}$ y $F_{1}$.

$Y$ añade un sentido más:

Un paso de argumento (válido) es analfíico si la información transmitida por la conclusión no es mayor que la información transmitida por las premisas ${ }^{7}$. Por otra parte, distingue la información superficial de la información profunda. La información profunda es el conjunto de todas las consecuencias lógicas de

${ }^{7}$ Cfr. Hintikka (1976): 175-6. 
una afirmación dada; la información superficial es el conjunto de sus consecuencias hasta un nivel de análisis prefijado. En algunos argumentos, la información superficial ofrecida en las premisas es suficiente para alcanzar la conclusión.

Sea, por ejemplo, el argumento: "Todas las ballenas son mamíferos y no todos los animales son mamíferos; luego no todos los animales son ballenas". Una esquematización lógica de este argumento es la siguiente:

P: ser ballena

$$
\forall \mathrm{x}(\mathrm{Px} \rightarrow \mathrm{Qx})
$$

Q: ser mamífero

$$
\frac{\neg \forall \mathrm{x}(\mathrm{Rx} \rightarrow \mathrm{Qx})}{\neg \forall \mathrm{x}(\mathrm{Rx} \rightarrow \mathrm{Px})}
$$

$\mathrm{R}$ : ser animal

Podemos concebir la primera premisa sin objetos y con independencia de lo que signifique "P» y lo que signifique "Q", por ejemplo asi:

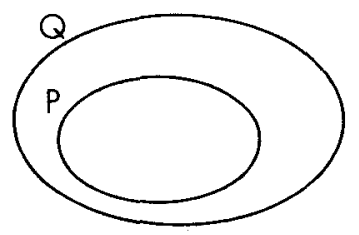

La segunda premisa, en cambio, tenemos que representarla así:

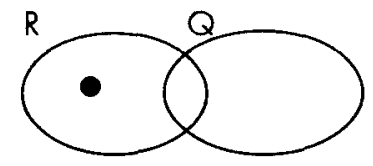

concibiendo algún individuo $R$ que no es $Q$.

Con esta intuición determinada en el conjunto $\mathrm{R}$, podemos unir las dos premisas y extraer la conclusión:

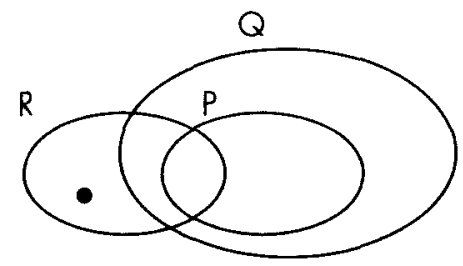

$\therefore$ Algún $\mathrm{R}$ no es $\mathrm{P}$. 
Pero no todos los esquemas deductivos son tan sencillos como éste. Si entendemos "no decir en la conclusión nada que no haya sido dicho ya en la premisas" en relación con la información profunda, tenemos que decir que no disponemos de medios para medirlo en todos los casos, pues la lógica de Primer Orden es no decidible; pero si nos referimos a una información superficial, muchos de nuestros argumentos válidos son sintéticos. Todas las verdades lógicas son tautologías profundas, pero no todas las verdades de la lógica de Primer Orden son tautologías superficiales.

Sin entrar en el lenguaje técnico utilizado por Hintikka para asociar grados de profundidad y pesos de información distribuida en cada grado, mediante un análisis que consiste en una sucesiva transformación de las fórmulas en sus formas normales disyuntivas, podemos presentar la idea de que algunas pruebas de la lógica cuantificacional requieren el uso de intuiciones mediante el cálculo clásico de la tablas analíticas o árboles semánticos. Este método mecánico nos permite comprender con relativa facilidad las dos principales ideas de Hintikka en relación con este tema: 1) la progresiva información superficial que el análisis nos va ofreciendo hasta llegar a igualarse con la información profunda, y 2) la necesidad de recurrir a la construcción de nuevos objetos para examinar las posibilidades de verdad en cada nivel de análisis. Es decir, en los argumentos válidos la conclusión no ofrece nueva información profunda, pero sí una superficial en algunos casos, y dicha información ganada es objetiva y no meramente psicológica.

Tomemos como ejemplo el siguiente esquema argumental:

$$
\begin{aligned}
& \forall \mathrm{x} \forall \mathrm{y} \forall \mathrm{z}(\mathrm{Ryz} \rightarrow(\mathrm{Qxz} \rightarrow \mathrm{Px})) \\
& \forall \mathrm{x} \forall \mathrm{y}(\neg \mathrm{Px} \wedge \mathrm{Rxy}) \\
& \neg \exists \mathrm{x} \exists \mathrm{y} \text { Qxy }
\end{aligned}
$$


Podemos tratar de probar la conclusión a partir de las premisas mediante las reglas del cálculo de deducción natural, por ejemplo, o bien tratar de probar que las premisas son incompatibles con la negación de la conclusión, es decir, que no puede darse el caso de que siendo verdaderas las premisas, la conclusión no lo sea, ese supuesto arroja una contradicción, el conjunto $\{\forall x \forall y \quad \forall z$ (Ryz $\rightarrow(\mathrm{Qxz} \rightarrow \mathrm{Px})), \forall \mathrm{x} \forall \mathrm{y}(\neg \mathrm{Px} \wedge \mathrm{Rxy}), \exists \mathrm{x} \exists \mathrm{y} \mathrm{Qxy}$ es contradictorio.

\begin{tabular}{|c|c|c|c|c|c|}
\hline$\sqrt{1}$ & $\forall x \forall y$ & $\forall z(R y$ & $\rightarrow(Q x z \rightarrow P x)) \wedge \forall x \forall y(\neg)$ & $x \wedge R(x y)$ & $\exists x \exists y Q x$ \\
\hline a,b & 2 & $\forall \mathbf{x} \forall \mathbf{y}$ & $\forall z\left(R_{y z} \rightarrow\left(Q x z \rightarrow P_{x}\right)\right)$ & $\alpha 1$ & \\
\hline a,b & 3 & $\forall \mathbf{x} \forall \mathbf{y}$ & $(\neg P x \wedge R x y)$ & $\alpha 1$ & \\
\hline$\sqrt{ }$ & 4 & $\exists \mathrm{x} \exists \mathrm{y}$ & $2 x y$ & $\alpha 1$ & \\
\hline $\mathbf{a}$ & 5 & $\forall y \quad \forall z$ & $(\mathrm{Ryz} \rightarrow(\mathrm{Qaz} \rightarrow \mathrm{Pa}))$ & $\gamma 2$ & \\
\hline $\mathbf{a}$ & 6 & $\forall z\left(R_{a}\right.$ & $\rightarrow(\mathrm{Qaz} \rightarrow \mathrm{Pa}))$ & $\gamma 5$ & \\
\hline$\sqrt{ }$ & 7 & $\mathrm{Raa} \rightarrow$ & $(\mathrm{Qaa} \rightarrow \mathrm{Pa})$ & $\gamma 6$ & \\
\hline $\mathbf{a}$ & 8 & $\forall \mathbf{y}(\neg)$ & a $\wedge$ Ray) & y3 & \\
\hline$\sqrt{ }$ & 9 & $\neg \mathrm{Pa} \wedge$ & Raa & $\gamma 8$ & \\
\hline & 10 & $\neg \mathrm{Pa}$ & & $\alpha 9$ & \\
\hline & 11 & Raa & & $\alpha 9$ & \\
\hline 12.1 & $\begin{array}{l}\neg \mathrm{Raa} \\
{ }_{\text {(11) }}\end{array}$ & & $\begin{array}{l}\sqrt{ } 12.2 \mathrm{Qaa} \rightarrow \mathrm{Pa} \\
13.21 \neg \mathrm{Qaa}\end{array}$ & $\begin{array}{l}\beta 7 \\
13.22 \mathrm{~Pa} \\
*(10)\end{array}$ & $\beta 12.2$ \\
\hline & $\sqrt{ }$ & $\begin{array}{l}14.21 \\
15.21\end{array}$ & $\begin{array}{l}\text { ヨy Qby } \\
\text { Qbc }\end{array}$ & $\begin{array}{l}84 \\
\delta 14.21\end{array}$ & \\
\hline & b & 16.21 & $\forall \mathrm{y} \forall \mathrm{z}(\mathrm{Ryz} \rightarrow(\mathrm{Qbz} \rightarrow \mathrm{Pb}))$ & $\gamma 2$ & \\
\hline & c & 17.21 & $\forall z(R b z \rightarrow(Q b z \rightarrow P b))$ & $\gamma 16.21$ & \\
\hline & $\sqrt{ }$ & 18.21 & $\mathrm{Rbc} \rightarrow(\mathrm{Qbc} \rightarrow \mathrm{Pb})$ & $\gamma 17.21$ & \\
\hline & c & 19.21 & $\forall \mathrm{y}(\neg \mathrm{Pb} \wedge \mathrm{Rby})$ & $\gamma 3$ & \\
\hline & $\sqrt{ }$ & 20.21 & $\neg \mathrm{Pb} \wedge \mathrm{Rbc}$ & $\gamma 19.21$ & \\
\hline & & 21.21 & $\neg \mathrm{Pb}$ & $\alpha 20.21$ & \\
\hline & & 22.21 & $\mathrm{Rbc}$ & $\alpha 20.21$ & \\
\hline 23.211 & $\neg$ Rbc & & $\begin{array}{c}\sqrt{ } 23.212 \mathrm{Qbc} \rightarrow \mathrm{Pb} \\
24.2121 \neg \mathrm{Qbc} \\
\cdot(15.21)\end{array}$ & $\begin{array}{c}\beta 18.21 \\
122 \mathrm{~Pb} \\
\cdot(21.21)\end{array}$ & $\beta 23.212$ \\
\hline
\end{tabular}

Hemos analizado, en primer lugar, las dos premisas (líneas 1-13), con un dominio mínimo, en el que hay un único objeto, y hemos visto que en una de las ramas se presenta una situación donde las dos premisas resultan verdaderas (13.21). A continuación hemos investigado en esta rama abierta la posibilidad 
de la falsedad de la conclusión. Para ello, nos hemos visto obligados a introducir dos objetos más (nuevos) en el mundo donde las premisas son verdaderas.

Lo que se sigue necesariamente de esas premisas es que ningún objeto tiene la relación (diádica) $Q$ con ningún objeto; para alcanzar esta conclusión hemos ensayado las posibilidades de verdad de $\exists \mathrm{x}$ ヨy Qxy «determinando el concepto en la intuición a priorin, como diría Kant, es decir, poniendo objetos nuevos relacionados mediante $Q$.

A medida que avanza el desarrollo del árbol, vamos ganando información superficial en el sentido de que el análisis nos va proporcionando situaciones posibles, mundos o estados de cosas donde podrían resultar verdaderas las premisas y falsa la conclusión. Cada rama del árbol nos va presentando una descripción que nos indica qué tipos de objetos podemos encontrar en el espacio (lógico) en el que la fórmula o conjunto de fórmulas resulta satisfecha. También nos va indicando qué tipo de objetos podemos encontrar en un examen posterior. Los distintos mundos descritos en cada rama y cada nivel (línea del árbol) tienen asociado un grado de creencia. A medida que avanzamos, según vamos desarrollando nuevas líneas, se van eliminando posibilidades (ramas cerradas por contener contradicción), los grados de creencia se van concentrando en los que permanecen como consistentes (en las ramas que siguen abiertas en el árbol) y, de este modo, se va reduciendo la incertidumbre, la información superficial va creciendo hasta igualarse (en el último grado) con la información profunda.

Esos mundos imposibles estaban prohibidos ya en la formula de la primera línea, pero en forma de información profunda. ¿Es, después de todo, un avance meramente psicológico? No, no se trata de no haber puesto la suficiente atención a la hora de pensar el significado de las premisas. Es un avance sintético aunque a priori en el sentido de Kant: para obtener la conclusión necesitamos el concurso de la sensibilidad (a priori). Mundos posibles en el grado n se desglosan en mundos posibles y mundos imposibles en el grado $\mathrm{n}+1$. Cuando alcanzamos la forma normal completa de la fórmula o el conjunto de fórmulas, encontramos qué posibilidades permanecen y cuáles han sido excluidas definitivamente. En este sentido, algunas deducciones, siendo válidas, proporcionan conocimiento superficial nuevo. La explicitación de una contradicción contenida en un conjunto de afirmaciones exige el ensayo con objetos puestos $a$ priori en la intuición. Tanto en el cálculo de validación de argumentos mediante deducción natural como en el cálculo de contraejemplos mediante árboles semán- 
ticos, la regla que permite eliminar un cuantificador existencial obliga siempre a introducir objetos nuevos en nuestro discurso. Cierto que la contradicción encontrada mediante el análisis estaba contenida ya el conjunto de partida, pero como las plantas están contenidas en las semillas, no como las vigas están contenidas en una casa. La necesidad de determinar conceptos en la intuición es, siguiendo tanto a Kant como a Frege, una condición suficiente para que todas aquellas pruebas en las que es necesario aplicar la regla de eliminación del existencial sean consideradas sintéticas.

"Los juicios sintéticos a priori son posibles cuando las condiciones formales de la intuición a priori (...) son referidas por nosotros a un conocimiento de experiencia posible en general y decimos: las condiciones de posibilidad de la experiencia en general son al mismo tiempo condiciones de la posibilidad de los objetos de la experiencia» (Kant, 1982:105).

Algunos pasos inferenciales son analíticos, otros son sintéticos. El que no pensemos la conclusión al pensar las premisas no se debe en todos los casos a que no prestemos la suficiente atención, sino al hecho de que la conclusión sólo puede ser alcanzada mediante el examen de individuos que no figuran en las premisas. Este cálculo provisional, con nombres de individuos que han de cumplir ciertos requisitos, y que el lógico hace para alcanzar la conclusión, es como el borrador que hace el geómetra dibujando una figura que cumpla las características dadas en las premisas. Por esto, podemos decir que la lógica cuantificacional (al menos aquella parte que depende de la regla de eliminación del cuantificador existencial) es constructiva, ampliativa, sintética.

Creo que Frege asumió con mucha rapidez la idea de la analiticidad de la lógica y quiso demostrar que la aritmética no era sino lógica desarrollada y, por tanto, también analítica. En una línea kantiana, podriamos decir que los juicios lógicos son sintéticos cuando dependen de nociones matemáticas, cuando en su prueba necesitamos determinar cuántos objetos y de qué tipo debe haber como mínimo en el mundo. 


\section{Conclusiones}

No es cierto que al conocer la verdad de un conjunto de afirmaciones podemos conocer, sin recurso a la intuición, todas sus consecuencias lógicas.

Si la geometría es sintética a priori porque proporciona nuevo conocimiento, que no proviene de la experiencia, sino únicamente de las condiciones formales de los fenómenos, de la forma de la sensibilidad, tendremos que decir que también la lógica formal es sintética a priori, ya que algunas de sus verdades dependen de la determinación de conceptos en la sensibilidad, de la construcción de casos determinados a priori. Un paso inferencial lógico (válido) es sintético cuando en su prueba necesitamos introducir nuevos individuos para hacer cálculos auxiliares. Esto ocurre siempre que necesitamos eliminar un cuantificador existencial.

Algunos cálculos no pueden darse sin espacio-tiempo, aunque la verdad logica es atemporal. $\mathrm{Al}$ igual que el geómetra pone una figura con tales y tales características en la intuición, el lógico construye una situación posible con tales y tales objetos, para investigar la validez de un argumento, pone un objeto en la intuición y no extrae de él ninguna propiedad, sino las que se siguen necesariamente de las que él mismo ha puesto.

Las salidas neokantianas ante esta situación deberían ser, o bien considerar que la parte sintética de la lógica ya no es propiamente lógica general, sino una ciencia especial, similar a la matemática, o bien afirmar que no todas las leyes de la lógica formal son leyes formales del pensar, sino únicamente las analíticas; sería muy discutible, por ejemplo, aceptar que el principio de monotonía, de la lógica clásica, es una condición necesaria de todo uso del entendimiento.

La lógica de Primer Orden es a priori, tautológica profunda, pero ampliativa superficial, constructiva, sintética.

\section{Bibliografía}

DeAÑo, A. (1980): Las concepciones de la Lógica, Taurus, Madrid.

DUMmETT, M. (1978): "The Justification of Deduction», en Truth and Other Enigmas, Duckworth, Londres (trad.: "Justificación de la deducción", La verdad y otros enigmas, F. C. E., México, 1990). 
FrEGE, G. (1884): Die Grundlagen der Arithmetik, Breslau, Koebner (trad.: Fundamentos de la aritmética, Laia, Barcelona, 1973).

FreGE, G. (1893, 1903): Grundgesetze der Arithmetik, 2 tomos, H. Pohle, Jena (trad. del prólogo y la introducción en Estudios sobre semántica, Ariel, Barcelona, 1984: 12176).

FREGE, G. (1969): Nachgelassene Schriften, F. Meiner Verlag, Hamburg (trad. inglés: Posthumous Writings, Basil Blackwell, Oxford, 1979).

HinTIKKA, J. (1973): Logic, Language-Games and Information, Oxford Univ. Press, Oxford (trad.: Logica, juegos de lenguaje e información, Tecnos, Madrid, 1976).

KANT, I. (1781-7): Kritik der reinen Vernunft, Riga (trad.: Crítica de la Razón Pura, Editorial Porrúa, México, 1982).

KANT, I. (1.800): Jäsche Logik, ed. de Jäsche, en Kant's gesammelte Schriften, De Gruyter, Berlín, 1902- :vol. IX (trad. en: Lógica. Un manual de lecciones. Acompañada de una selección de Reflexiones del legado de Kant, Akal, Madrid, 2000).

KANT, I. (1882-4): Reflexionen Kants zur kritischen Philosophie. Aus Kant bandschriftlichen Aufzeichnungen, 2 vols., Leipzig (trad. parcial: Reflexiones, en Kant 2000).

KRIPKE, S. (1982): Wittgenstein on Rules and Private Language. An Elementary Exposition, Basil Blackwell, Oxford (trad.: Wittgenstein: Reglas y lenguaje privado, UNAM, México, 1989).

MacFarlane, J. (2002): "Frege, Kant, and the Logic in Logicism», The Philosophical Review 111: 25-65.

QUINE, W.v.O. (1951): "Two Dogmas of Empiricism», Philosophical Review 60: 20-43 (trad.: «Dos dogmas del empirismo», Desde un punto de vista lógico, Orbis, Barcelona, 1984).

WitTgenstein, L. (1922): Tractatus logico-philosophicus, Routledge \& Kegan Paul, Londres (trad.: Tractatus logico-philosophicus, Alianza, Madrid, 1987).

WitTGenstein, L. (1978): Remarks on the Foundations of Mathematics, Basil Blackwell, Oxford (trad.: Observaciones sobre los fundamentos de la matemática, Alianza, Madrid, 1987). 